\title{
Rmr180093R2
}

Fascicule vert

Article original

\section{Les patients de la cohorte IDEAL : une photographie de l'asthme sévère en France}

\section{Patients in the IDEAL cohort: a snapshot of severe asthma in France}

C. Taillé ${ }^{1}$, Christophe Pison ${ }^{2}$, Cécilia Nocent ${ }^{3}$, Gilles Devouassoux ${ }^{4}$, A Prud'homme $^{5}$, A. Gruber ${ }^{6}$, N. Gunsoy ${ }^{7}$, F. Albers ${ }^{8}$

${ }^{1}$ Service de Pneumologie, DHU FIRE, Hôpital Bichat, AP-HP, Paris, France et INSERM U1152, Université Paris Diderot, Labex Inflamex, 75018, Paris, France.

${ }^{2}$ CHU Grenoble Alpes, Service Hospitalier Universitaire Pneumologie Physiologie, Pôle Thorax et Vaisseaux, Grenoble, Université Grenoble Alpes, France

${ }^{3}$ Service de Pneumologie, $\mathrm{CH}$ de la côte Basque, Bayonne, France

${ }^{4}$ Service de Pneumologie, Hôpital de la Croix Rousse, Hospices Civils de Lyon, Lyon, France

${ }^{5}$ Service de Pneumologie, CH de Bigorre, Tarbes, Vic en Bigorre, France

${ }^{6}$ GSK, Rueil-Malmaison, France

${ }^{7}$ GSK, Stockley Park, Uxbridge, UK

${ }^{8}$ GSK, Research Triangle Park, NC, USA.

\section{Titre court :}

Asthme sévère en France : cohorte IDEAL

\section{Auteur-correspondant :}

Camille Taillé

Service de Pneumologie, Hôpital Bichat, APHP, Paris, France.

00331402568 63. camille.taille@aphp.fr

Reçu le : 01.05 .18

Accepté le : 18.06 .18

\section{Déclaration de liens d'intérêts :}

Au cours de ces cinq dernières années, Camille Taillé a reçu des honoraires pour des expertises et des présentations orales, a été investigateur pour des essais et a été invitée à des congrès des laboratoires AstraZeneca, GSK, Teva, Novartis, Roche, Sanofi, Boehringer Ingelheim et Chiesi.

Christophe Pison a reçu des remboursements des frais d'inscriptions et déplacements à des réunions médicales et des honoraires pour des formations médicales des laboratoires GSK, Astra-Zeneca, Boehringer Ingelheim, Teva et Chiesi.

Cécilia Nocent a reçu des honoraires des laboratoires GSK, Astra Zeneca, Teva, Chiesi, Mundipharma, Boehringer Ingelheim, Novartis et ALK.

Gilles Devouassoux a été consultant pour les laboratoires Novartis, Astra-Zeneca, GSK, Boehringer Ingelheim, Mundipharma, Vivisol, Sanofi, Chiesi, ALK et Teva. Il a reçu des remboursements de frais 
pour sa participation à des réunions médicales de GSK, Astra-Zeneca, Novartis Pharma, Chiesi, MSD, Takeda, AGIR à dom, Orkyn, Mundipharma, ALK, Stallergène, Boehringer Ingelheim et Teva, ainsi que des bourses de recherche de GSK, Novartis Pharma, MSD, Chiesi et AGIR à dom. Il a été investigateur pour des essais cliniques pour GSK, ALK, Novartis Pharma, Boehringer-Ingelheim, Vitalair, AB Science, Amgen, Lilly, Astra-Zeneca, Sanofi, Roche et Teva.

Anne Prud'homme a été consultante pour les laboratoires Roche et a apporté son expertise aux laboratoires Novartis, Teva, Mundipharma, Astra-Zeneca et GSK. Elle a reçu des honoraires pour des interventions ponctuelles pour Astra-Zeneca, ALK, Mundipharma, GSK, BIF et Teva. Elle a été invitée à des conférences et des congrès par Boehringer Ingelheim, Novartis, MundiPharma, GSK, ALK et Astra-Zeneca.

Alina Gruber est une employée de la filiale France de GSK, Necdet Gunsoy de la filiale UK et Frank Albers de la filiale USA.

\section{Financements}

Une aide à la rédaction, mise en forme, révision et bibliographie du manuscrit, ainsi que la collecte et l'intégration des commentaires des auteurs dans le manuscrit ont été fournies par Brigitte Bourdillat, $\mathrm{PhD}$, rédacteur médical indépendant, et financées par GSK. Cette étude a été financée par GSK (étude ID 201722 ; NCT02293265). 


\section{Résumé}

Introduction.- Cet article rapporte les résultats d'une analyse post-hoc contenant les données françaises de l'étude internationale IDEAL dont l'objectif était de décrire une cohorte récente d'asthmatiques sévères, l'impact de la maladie sur la qualité de vie des patients, ainsi que la population de patients éligibles à omalizumab, mépolizumab et reslizumab.

Méthodes. - Tout patient âgé $\geq 12$ ans avec un asthme sévère (GINA 4-5) était éligible.

Résultats.- Les 129 patients inclus dans cette analyse post-hoc, d'âge moyen de 53 ans, étaient majoritairement en surpoids et de sexe féminin (64\%) et avaient au moins une comorbidité (85\%). Plus d'un patient sur deux souffrait d'asthme depuis plus de 25 ans et était non-fumeur. La fonction respiratoire était modérément altérée. Un taux d'éosinophiles $\geq 150$ cellules/ $\mu 1$ était rapporté chez $66 \%$, $\geq 300$ cellules/ $\mu 1$ chez $34 \%$ et $\geq 500$ cellules/ $\mu 1$ chez $12 \%$ des patients. Un patient sur trois était traité par omalizumab et $24 \%$ recevaient des corticoïdes oraux en traitement de fond. L'asthme était mal contrôlé avec un impact négatif sur la qualité de vie (ACQ $\geq 1,5: 67 \%$ des patients). Dans la population, 40\%, $27 \%$ et $2 \%$ des patients étaient éligibles respectivement à omalizumab, mépolizumab et reslizumab.

Conclusions- Ces résultats montrent qu'il reste de nombreux patients asthmatiques sévères non contrôlés et non éligibles aux traitements biologiques disponibles. Ceci souligne le besoin d'innovation thérapeutique dans cette pathologie.

Mots-clés : asthme sévère, mépolizumab, omalizumab, étude observationnelle 


\section{Patients in the IDEAL cohort: a snapshot of severe asthma in France}

Introduction. This paper reports the French data from a post-hoc analysis of the international IDEAL study, which aimed to describe a recent cohort of patients with severe asthma, the impact of the disease on quality of life, as well as the population of patients eligible for treatment with omalizumab, mepolizumab and reslizumab.

Methods.- Eligible patients were $\geq 12$ years of age, with severe asthma (GINA steps 4 and 5).

Results.- A total of 129 patients were included in this post-hoc analysis. Their mean age was 53 years, the majority were overweight, they were mainly women (64\%) and had at least one medical comorbidity (85\%). More than half had suffered from asthma for more than 25 years and were nonsmokers. Lung function was moderately impaired. Blood eosinophil count was $\geq 150$ cells $/ \mu \mathrm{L}$ in $66 \%$ of patients, $\geq 300$ cells $/ \mu \mathrm{L}$ in $34 \%$ of patients, and $\geq 500$ cells $/ \mu \mathrm{L}$ in $12 \%$ of patients. One out of three patients was currently treated with omalizumab and $24 \%$ had maintenance oral corticosteroids. Asthma was poorly controlled with a negative impact on quality of life (ACQ $\geq 1.5$ ) in $67 \%$ of patients. In this population $40 \%$ of patients were eligible for omalizumab, $27 \%$ for mepolizumab and $2 \%$ for reslizumab.

Conclusions.- These findings show that a considerable proportion of patients with severe asthma remain uncontrolled and are not eligible for any of the available biological treatments. This underlines the need for therapeutic innovations in this disease.

Key-words: severe asthma, mepolizumab, omalizumab, observational study. 


\section{Introduction}

L'asthme sévère défini selon les niveaux 4-5 GINA (Global Initiative for Asthma) concerne 3,6 à 10\% des asthmatiques [1-3]. Ceux-ci sont caractérisés par un âge plus élevé, davantage de comorbidités et d'exacerbations, un recours plus important aux soins spécialisés, comparativement à une population d'asthmatiques persistants non sévères dans une vaste étude observationnelle menée chez 25935 patients [4].

Sur le plan clinique, l'hétérogénéité de l'asthme sévère contribue à la variabilité de la réponse aux traitements. Différents phénotypes cliniques ont été identifiés dans l'objectif de personnaliser la prise en charge [5-8]. L'asthme sévère allergique est induit par des réactions inflammatoires médiées en grande partie par les IgE [2,9]. Un autre phénotype d'asthme sévère se caractérise par une inflammation éosinophilique des voies aériennes [2,10-11]. Ces deux phénotypes ne sont pas exclusifs, puisque les patients atopiques présentent fréquemment une inflammation éosinophilique des voies aériennes. Ces

deux mécanismes inflammatoires sont des cibles de traitement par des anticorps monoclonaux : l'omalizumab, qui bloque les IgE circulantes, le mépolizumab et le reslizumab qui diminuent le taux d'éosinophiles en neutralisant l'IL-5 [2,12-15]. Les critères permettant d'identifier les patients asthmatiques sévères cibles de chacun des traitements d'anticorps monoclonaux actuels ont été définis par les essais cliniques de phase 3 [6,16-19]. Toutefois, peu d'études se sont intéressées à la population de patients qui pourrait bénéficier davantage de l'un ou l'autre des différents traitements biologiques et à estimer l'éligibilité à chacun d'eux.

Dans ce contexte, une étude internationale, l'étude IDEAL (GSK NCT02293265), a été menée dans six pays incluant la France (Australie, Canada, France, Allemagne, Royaume-Uni et Etats-Unis) pour décrire, au sein d'une cohorte de patients asthmatiques sévères, la population de patients remplissant les critères d'éligibilité d'un traitement par anti-IgE (omalizumab) selon les critères locaux de prescription et de deux traitements par anti-IL5 (mépolizumab et reslizumab) selon les critères d'inclusion/exclusion des études cliniques, ces deux produits étant en cours d'enregistrement lors de la mise en place de 
l'étude. Les objectifs secondaires de l'étude IDEAL étaient de décrire les caractéristiques cliniques de la population de l'étude et l'impact de la maladie sur la qualité de vie des patients.

Cet article présente les résultats d'une analyse post-hoc contenant les données de la cohorte française, qui représentait 19\% de la cohorte mondiale de l'étude IDEAL [16].

\section{Patients et méthodes}

L'étude IDEAL est une étude épidémiologique transversale menée auprès de médecins pneumologues libéraux ou exerçant en centres hospitaliers et prenant en charge régulièrement des patients asthmatiques sévères. En France, 17 centres ont accepté de participer à l'étude et 15 ont inclus des patients. L'étude s'est déroulée du 8 décembre 2014 au $1^{\text {er }}$ mai 2015.

Les patients étaient sélectionnés lors d'une visite de présélection (V0). La durée de l'étude était limitée à une seule visite (V1). Les deux visites V0 et V1 pouvaient avoir lieu le même jour.

Aucun traitement n'a été administré durant l'étude. La méthodologie complète de l'étude est détaillée dans l'article récemment publié sur la cohorte mondiale [16].

\section{Population éligible pour la cohorte IDEAL}

Tous les patients âgés d'au moins 12 ans le jour de la visite étaient éligibles pour l'étude s'ils présentaient un asthme sévère nécessitant, depuis au moins un an, un traitement par fortes doses de corticostéroïdes inhalés (CSI) associé à l'un au moins des traitements suivants : béta2-agoniste inhalé de longue durée d'action (LABA), antileucotriène, théophylline, omalizumab ou corticostéroïdes systémiques en traitement chronique pendant au moins six mois au cours de l'année précédente.

Les patients ayant participé à un essai clinique interventionnel sur l'asthme dans les 12 mois qui précédaient la visite n'étaient pas éligibles.

\section{Recueil des données}

Lors de la visite, les données démographiques, les antécédents médicaux, l'histoire de l'asthme, les antécédents d'intubation, les facteurs de risque cardiovasculaire et les traitements reçus antérieurement durant les 12 derniers mois ont été collectés. 
Lors de cette visite, les patients devaient remplir les auto-questionnaires suivants :

- Questionnaire de contrôle de l'asthme en 5 items (ACQ-5) dont l'évaluation concernait la semaine précédant la visite. Les scores s'échelonnent de 0 (totalement contrôlé) à 6 (sévèrement non contrôlé).

- Questionnaire respiratoire de Saint Georges (SGRQ) conçu en 50 questions pour mesurer la qualité de vie au cours des 3 derniers mois [20]. Les scores s'échelonnent de 0 (pas de retentissement) à 100 (retentissement important).

- Questionnaire standardisé de qualité de vie en 32 questions (AQLQ) pour évaluer l'impact physique et émotionnel de la maladie au cours des deux dernières semaines [21]. Les scores s'échelonnent de 0 (pire qualité de vie) à 6 (meilleure qualité de vie).

- Questionnaire standardisé de qualité de vie européenne (EQ-5D-5L) structuré en deux parties. La première partie comporte 5 questions permettant d'évaluer la mobilité, les activités quotidiennes, les soins autonomes, les douleurs/malaises et l'inquiétude/ dépression dont le score varie de 0 (pire état) à 1 (meilleur état). Le questionnaire se termine par une échelle visuelle analogique (EQ-EVA) dont le score varie de 0 (pire état de santé) à 100 (meilleur état de santé imaginable) à [22].

- Questionnaire du Work Productivity and Activity Impairment (WPAI) [23]. Les pourcentages les plus élevés de WPAI indiquent une limitation des activités plus importante et une productivité diminuée au cours de la semaine précédente.

- Asthma symptom utility index (ASUI) comportant 10 items pour évaluer la fréquence et la sévérité de quatre symptômes d'asthme (toux, respiration sifflante, essoufflement, réveil nocturne) et les effets secondaires des traitements anti-asthmatiques au cours des deux dernières semaines [24]. Les scores totaux s'échelonnent de 0 (pires symptômes) à 1 (aucun symptôme). L'évaluation clinique comportait, pour tous les patients, un examen médical et pour ceux n'ayant pas eu d'exploration fonctionnelle respiratoire dans les 12 derniers mois, une spirométrie et un test de réversibilité. Une numération-formule sanguine et un dosage des $\operatorname{IgE}$ sériques (totales et spécifiques: acariens [Dermatophagoides farinae, D. pteronyssinus], poils de chats, chiens, moisissures et pollens 
(arbres et graminées) étaient réalisés lors de la visite. L'atopie était définie par la présence d'un test

RAST positif. Les analyses ont été effectuées par un laboratoire central (Quest Middlesex, UK).

\section{Critères d'éligibilité aux traitements biologiques}

Les critères d'éligibilité aux trois traitements biologiques, omalizumab, mépolizumab et reslizumab sont détaillés dans le Tableau 1. Tout patient traité par omalizumab au moment de la visite a été automatiquement qualifié en patient éligible à l'omalizumab, mais les critères de prescription initiaux n'étaient pas recueillis, puisque le traitement n'avait pas toujours été initié par le centre incluant le patient dans l'étude.

\section{Analyse statistique des données}

L'analyse des données est uniquement descriptive. Les données quantitatives sont présentées avec leur moyenne \pm écart-type pour les variables continues après vérification de leur distribution normale, sinon leur médiane avec leur $25^{\mathrm{e}}$ et $75^{\mathrm{e}}$ percentile. Les données qualitatives sont présentées avec leur effectif et pourcentage. Tous les patients asthmatiques sévères pour lesquels des données permettaient l'évaluation de l'éligibilité aux traitements biologiques ont été inclus dans la population totale de la cohorte française. L'analyse a été faite sur deux sous-groupes, l'un composé des patients ne recevant pas omalizumab au moment de la visite et l'autre de patients en cours de traitement par omalizumab.

Le logiciel SAS 9.2 (SAS Institute, Cary, NC) a été utilisé pour l'analyse statistique.

\section{Résultats}

La population totale de la cohorte française se composait de 129 patients (Fig. 1) dont 43 patients (33\%) recevant de l'omalizumab au moment de l'étude.

\section{Caractéristiques de la cohorte}

Les caractéristiques des patients sont détaillées dans le Tableau 2. Les patients avaient un âge moyen de 53 ans, étaient majoritairement des femmes (64\%) et avaient un indice de masse corporelle (IMC) supérieur à $25 \mathrm{~kg} / \mathrm{m}^{2}$. Plus d'un patient sur deux (59\%) n'avait jamais été fumeur et la majorité des autres $(38 / 53,74 \%)$ était composée d'anciens fumeurs. La majorité des patients (87\%) présentait au 
moins une comorbidité. Parmi les 88 patients (68\%) présentant une symptomatologie respiratoire, 53 souffraient de rhinite allergique ou de rhume des foins, 22 de polypes nasaux, et 19 de sinusite. Parmi les 56 patients présentant un trouble du métabolisme, 34 étaient obèses, 22 avaient de l'ostéoporose et 14 du diabète. Parmi les 42 patients souffrant de troubles du système nerveux, 27 présentaient des troubles du sommeil, 25 souffraient d'anxiété et 13 de dépression. La majorité des patients présentant un trouble cardiovasculaire souffrait d'une hypertension (34/40). Parmi les 59 patients présentant une autre comorbidité que les précédentes, 46 patients souffraient d'un reflux gastro-œsophagien, 17 présentaient une sensibilité à l'aspirine et 6 souffrait d'un eczéma. Un patient sur deux (53,5\%) était atopique, selon les critères de l'étude (un test RAST positif) Le taux d'IgE totales était inférieur à 30 kU/L chez 17\% des patients.

\section{Antécédents d'asthme}

Plus de la moitié des patients (55\%) souffraient d'asthme depuis plus de 25 ans et presque un sur deux (47\%) avait des antécédents familiaux d'asthme. Plus d'un patient sur trois (36\%) a présenté au moins deux exacerbations nécessitant le recours aux corticoïdes et/ou une consultation aux urgences et/ou une hospitalisation, et un patient sur huit $(12 \%)$ a présenté au moins une exacerbation ayant nécessité une hospitalisation. Environ 10\% des patients avaient dû être intubés pour une exacerbation dans les 12 derniers mois. Les caractéristiques des patients étaient similaires, qu'ils reçoivent ou non un traitement par omalizumab au moment de l'étude, et sont détaillées dans le Tableau 2.

\section{Fonction respiratoire}

Le volume expiratoire maximal par seconde (VEMS) moyen était proche de $70 \%$ de la valeur théorique avec une réversibilité moyenne de 9\%, sans différence selon qu'un traitement par omalizumab était reçu au moment de l'étude ou non (Tableau 2).

\section{Taux d'éosinophiles sanguins}

Pendant les 12 mois précédant la visite, le taux d'éosinophiles sanguins a été renseigné chez moins d'un patient sur deux $(\mathrm{n}=62,48 \%)$ et était supérieur ou égal à 300 cellules/ $\mu 1$ chez plus de la moitié des patients ayant un taux renseigné (34/62). Lors de la visite, un patient sur trois, qu'il soit traité ou non par 
omalizumab, avait un taux d'éosinophiles supérieur ou égal à 300 cellules/ $\mu 1$ et $12 \%$ un taux d'éosinophiles supérieur ou égal à 500 cellules/ $\mu 1$ (Tableau 2).

\section{Traitement par corticoïdes oraux}

Lors de la visite, presqu'un quart des patients $(n=31,24 \%)$ recevait des corticoïdes oraux en traitement de fond (Tableau 2). Parmi les 9 patients qui avaient reçu des corticoïdes oraux en traitement de fond pendant l'année précédente, le traitement avait été arrêté pour les raisons suivantes: asthme non contrôlé ( $\mathrm{n}=2)$, décision du médecin estimant que l'asthme était contrôlable sans corticoïdes oraux $(n=6)$, effets indésirables $(n=2)$, contre-indication $(n=1)$, autres $(n=1)$. De nombreux patients $(n=89$, 69\%) n'avaient jamais reçu de corticoïdes en traitement de fond du fait d'une décision du médecin estimant que l'asthme était contrôlable sans corticoïdes oraux $(n=74)$, d'effets indésirables $(n=10)$, d'une contre-indication $(n=3)$ ou pour d'autres raisons $(n=10)$. Les patients traités par omalizumab lors de la visite étaient moins fréquemment traités par corticoïdes, que ce soit lors de la visite de l'étude ou au cours des 12 mois précédents.

\section{Traitement antérieur par omalizumab}

Un tiers des patients recevait de l'omalizumab au moment de l'étude. Leurs caractéristiques sont présentées dans le Tableau 2. Plus d'un patient sur trois (36\%) avait reçu un traitement par omalizumab au cours des 12 derniers mois précédant la visite V1 (Tableau 2). L'administration était mensuelle pour plus d'un patient sur deux (60\%). La durée médiane du traitement a été de 20 mois [1-73]. Les raisons de l'arrêt du traitement n'ont pas été précisées.

\section{Perception de la sévérité de la maladie et de l'impact sur la qualité de vie}

Les résultats rapportés dans les auto-questionnaires patients ont mis en évidence un mauvais contrôle de l'asthme avec $67 \%$ des patients ayant un score ACQ-5 $\geq 1,5$, et $15,5 \%$ avec un score ACQ-5<0,75. Les valeurs moyennes du score ACQ-5 dans la population française selon le traitement en cours par omalizumab sont rapportées dans le Tableau 2. La qualité de vie, mesurée, par 3 scores différents, était très altérée. 


\section{Eligibilité aux trois traitements biologiques}

Dans la population pour qui le taux d'éosinophiles était renseigné, plus d'un patient sur quatre était éligible au mépolizumab (27,1\%). L'éligibilité à l'omalizumab selon les critères d'éligibilité retenus, concernait plus d'un patient sur trois (40,3\%). Les patients éligibles au reslizumab ne représentaient que 2,3\%. Dans le sous-groupe de patients ne recevant pas de traitement par omalizumab au moment de l'étude, 29,1\% des patients étaient éligibles au mépolizumab et 10,5\% à l'omalizumab. Dans le sousgroupe de patients en cours de traitement par omalizumab, 23,3\% des patients étaient éligibles au mépolizumab. L'éligibilité aux trois traitements biologiques selon le traitement en cours par omalizumab est détaillée dans le Tableau 3. Parmi les 35 patients éligibles au mépolizumab, 17 patients étaient aussi éligibles à l'omalizumab dont 10 en cours de traitement.

Les caractéristiques des patients, selon leur éligibilité aux traitements, sont détaillées dans le Tableau 4.

\section{Discussion}

Les données épidémiologiques françaises dans l'asthme sévère sont rares. L'atout principal de l'étude IDEAL est de décrire une cohorte récente de patients présentant un asthme sévère en France et l'impact de la maladie sur la qualité de vie des patients, mais aussi de quantifier et caractériser les patients remplissant les critères d'éligibilité d'un traitement par omalizumab, mépolizumab et reslizumab.

\section{Données démographiques et cliniques}

Les données françaises montrent que la majorité des patients sont des femmes, d'âge moyen 53 ans, en surpoids, non-fumeurs, et souffrant d'asthme depuis plus de 25 ans. Ces données confirment les tendances observées dans différentes cohortes, européennes et américaines, avec une prépondérance de femmes et des patients en surpoids [25-31]. La proportion de fumeurs actuels dans notre cohorte française est similaire à celle trouvée dans une cohorte belge [30] avec plus d'un patient sur dix, mais deux fois plus élevée que celle trouvée dans le registre anglais (5,8\%) [27] et dans la cohorte américaine TENOR (4,3\%) [28]. Toutefois, il est intéressant de noter que la proportion d'anciens fumeurs trouvée dans notre cohorte $(29 \%)$ est similaire à celles trouvées dans la cohorte belge (BSAR, 31\%) [30], la 
cohorte et le registre anglais (25,5\% et $29,8 \%$, respectivement) [31,27] ainsi que dans la cohorte TENOR (32\%) [28]. Aujourd'hui, le sevrage tabagique constitue un des objectifs de la prise en charge de l'asthme, tout comme le traitement des comorbidités (rhino-sinusite chronique, polypes nasaux, anxiété et dépression, obésité, syndrome d'apnée du sommeil, reflux gastro-œsophagien) qui sont fréquentes chez les patients asthmatiques sévères et contribuent à un mauvais contrôle de l'asthme et à la survenue d'exacerbations [32,33]. Dans la cohorte belge BSAR, 49\% des patients présentaient une rhinosinusite et 36\% un reflux gastro-œsophagien [30]. Dans la cohorte IDEAL, la majorité des patients présentait au moins une comorbidité fréquente chez les asthmatiques sévères, telle que rhinite allergique, reflux gastro œsophagien, obésité, hypertension, troubles du sommeil, anxiété, polypose nasale, ostéoporose, diabète et dépression. Un patient sur deux était atopique, proportion concordante avec celle (57\%) rapportée dans le registre anglais [27], mais plus élevée (43\%) que celle du groupe d'asthmatiques sévères de la cohorte BIOAIR [29] et plus faible (70\%) que celle de la cohorte BSAR [30]. Plus d'un patient sur trois a présenté plus de deux exacerbations sous corticoïdes oraux, et/ou s'est rendu aux urgences et/ou a été hospitalisé, reflétant un mauvais contrôle de la maladie.

Le niveau d'obstruction des voies aériennes est similaire à celui retrouvé dans les cohortes BSAR [30], ENFUMOSA [26] et BIOAIR [29] et le registre anglais [27], mais plus faible que celui mis en évidence dans la cohorte américaine SARP chez les patients asthmatiques sévères [34].

Plus d'un patient sur deux de la cohorte IDEAL présentait un taux d'éosinophiles supérieur à 150 cellules/ $\mu \mathrm{l}$ et $34 \%$ des patients avaient un taux supérieur à 300 cellules/ $\mu \mathrm{l}$, et étaient donc potentiellement éligibles à un traitement anti-IL5. Ces résultats sont similaires à ceux trouvés dans la cohorte BSAR ou en population générale d'asthme dans la cohorte anglaise [30,31]. Un taux médian de 300 cellules/ $\mu 1$ a été retrouvé dans le registre anglais [27]. Toutefois, le taux d'éosinophiles peut être élevé, indépendamment de la sévérité de l'asthme [31].

\section{Fardeau de la maladie}

Au regard de la qualité de vie des patients inclus dans l'étude IDEAL, les résultats obtenus avec l'ensemble des auto-questionnaires utilisés confirment l'impact majeur de l'asthme sévère sur la vie de 
patients. Ils montrent un retentissement important sur le mode de vie, en particulier pour les capacités à effectuer les activités quotidiennes, illustrant ainsi le fardeau de la maladie chez des patients asthmatiques sévères en France. Les scores ACQ de l'étude IDEAL sont similaires à ceux trouvés dans les cohortes BIOAIR $(2,0 \pm 0,1)$, BSAR $(2,6 \pm 0,1)$ et COBRA $(2,1 \pm 1,4)[29,30,35]$. Des scores AQLQ et SGRQ similaires à ceux retrouvés dans l'étude IDEAL ont été mis en évidence dans les cohortes BSAR (AQLQ : 4,1 \pm 0,1) et BIOAIR (SGRQ : 45,9 $\pm 2,1$ ) [30,29]. Dans la récente étude française COBRA, le fardeau de la maladie se traduit par un coût médical direct de 8222 euros par an pour un patient asthmatique sévère [35]. De plus, les exacerbations de l'asthme restent un problème majeur dans la prise en charge et l'évolution des asthmatiques. Il a été montré qu'une exacerbation récente est l'indicateur le plus fiable d'une future exacerbation [28]. Dans l'étude IDEAL, 36\% des patients avaient présenté au moins deux exacerbations nécessitant le recours aux corticoïdes et/ou une consultation aux urgences et/ou une hospitalisation, et $24 \%$ recevaient des corticoïdes oraux en traitement de fond. Ces résultats sont comparables à ceux de la cohorte COBRA avec $31 \%$ des patients ayant eu des exacerbations sévères et $22 \%$ des patients traités par corticoïdes oraux durant au moins 6 mois [35]. La comparaison de la cohorte française de l'étude IDEAL avec la cohorte mondiale, montre que les patients français étaient plus nombreux à avoir des exacerbations sévères et à recevoir des corticoïdes en traitement de fond

\section{Prise en charge thérapeutique}

Dans la cohorte française de l'étude IDEAL, près d'un quart des patients recevaient une corticothérapie au long cours et plus d'un sur trois avait reçu un traitement par omalizumab au cours de l'année précédente. Le traitement par des corticoïdes oraux ainsi que les antécédents de traitement par omalizumab semblent moins fréquents dans la cohorte mondiale que dans la cohorte française [16]. De plus, $67 \%$ des patients ayant un asthme sévère ne recevaient pas de traitement par omalizumab dans la cohorte française. Parmi les patients non traités avec omalizumab, 57\% des patients n'étaient pas éligibles à l'un des produits biologiques évalués dans l'étude. Au niveau de la cohorte mondiale, le taux de patients non éligibles à l'un des trois traitements éligibles est encore plus considérable et varie de 
65\% en Australie/Canada/Etats-Unis à $76 \%$ en Europe [16]. Ces résultats mettent en évidence qu'il existe un besoin thérapeutique non couvert dans la population d'asthmatiques sévères non contrôlés et difficilement pris en charge par les traitements existants. Ce « trou » thérapeutique concerne notamment les patients n'ayant pas de marqueurs de la voie Th2 très élevés. Il existe quelques alternatives aux traitements par anticorps monoclonaux, comme les macrolides ou la thermoplastie bronchique, mais leur place respective dans la stratégie de prise en charge de l'asthme ne fait pas actuellement l'objet de recommandation de la part du GINA ou de la HAS (Haute Autorité de Santé). Les patients éligibles ou potentiellement répondeurs à ces traitements ne sont d'ailleurs pas définis. Des stratégies non médicamenteuses, comme l'amaigrissement pour les obèses, ou la réhabilitation respiratoire, font partie des éléments qui peuvent être discutés pour certains patients.

\section{Eligibilité aux différents traitements}

Il est intéressant pour le clinicien de pouvoir disposer de critères cliniques lui permettant de sélectionner les patients qui pourront bénéficier de l'un ou l'autre des traitements et ainsi de personnaliser le traitement pour chaque patient asthmatique sévère. L'étude internationale IDEAL s'inscrit dans l'aide à une démarche de médecine personnalisée. Ainsi, les données françaises montrent que plus d'un patient sur quatre (27\%) souffrant d'asthme sévère était éligible au mépolizumab selon les critères suivants : un taux d'éosinophiles sanguins $\geq 150$ cellules/ $\mu \mathrm{L}$ lors de la visite d'inclusion dans l'étude ou $\geq 300$ cellules/ $\mu 1$ dans les douze derniers mois et au moins deux épisodes d'exacerbations asthmatiques ayant nécessité un traitement par corticoïde oral, une venue aux urgences ou une hospitalisation dans l'année précédente. Toutefois, les taux d'éosinophiles sanguins définissant les critères d'éligibilité au mépolizumab en France ont évolué depuis la mise en place de l'étude IDEAL et ont été restreints par la Commission de Transparence en juin 2016 [36] à une valeur $\geq 300$ cellules/ $\mu 1$ dans les douze derniers mois et demandent à être confirmés dans une étude tenant compte de ces nouveaux critères d'éligibilité et incluant plus de patients. De plus, la proportion de patients présentant un asthme sévère éligible au reslizumab dans l'étude IDEAL selon les critères définis dans les études de phase III (Tableau 1) était faible $(<3 \%)$. Ces critères de sélection sont très différents de ceux proposés depuis par la HAS en juin 
2017 [37] , qui n'incluent pas de critère de réversibilité ni de score de contrôle. Ces critères peuvent expliquer la faible proportion de patients éligibles au reslizumab : un quart des patients avait une réversibilité $>12 \%$ et moins d'un quart avait un taux d'éosinophiles $>400$ cellules $/ \mu 1$. Il est probable que l'application des nouveaux critères modifierait sensiblement cette proportion de patients éligibles.

\section{Limites de l'étude}

Tout d'abord, un biais dans la sélection des patients, favorisant les moins bien contrôlés ou les patients en échec de traitement, ne peut être écarté, lié aux choix des investigateurs, prenant en charge régulièrement des asthmes sévères. Ceci est important pour expliquer que les patients traités par omalizumab ont autant d'exacerbations et sont autant traités par des corticoïdes oraux que les non traités. Ceci ne préjuge pas de l'effet de l'omalizumab, dans la mesure où le niveau de contrôle avant le début du traitement n'est pas connu. D'autre part, aucune donnée sur l'observance ou le contrôle des comorbidités par exemple, qui peuvent contribuer également au mauvais contrôle, n'est disponible. Enfin, l'interprétation des résultats sur l'éligibilité est limitée du fait des petits effectifs dans les différents sous-groupes et du fait du nombre important de données manquantes sur l'éosinophilie, ainsi que des critères d'éligibilité aux traitements disponibles à la mise en place de l'étude. Ces données nécessitent d'être confirmées sur des effectifs plus importants et en prenant en compte les critères d'éligibilité actuels définis par la HAS.

\section{Conclusion}

La cohorte IDEAL décrit une population française d'asthmatiques sévères et permet de disposer de données épidémiologiques récentes sur les caractéristiques actuelles de cette population. Cette population est majoritairement composée de femmes, de patients en surpoids, non-fumeurs, atopiques, souffrant d'asthme depuis plus de 25 ans, avec des antécédents familiaux d'asthme et présentant au moins une comorbidité. Les résultats des auto-questionnaires mettent en évidence un mauvais contrôle de l'asthme et un impact sévère sur la qualité de vie. Lors de la visite de l'étude, un quart des patients était traité par des corticoïdes oraux, et un tiers par omalizumab. Plus d'un patient sur trois et plus d'un 
sur quatre était éligible respectivement à omalizumab et au mépolizumab. Cette étude montre qu'il reste encore de nombreux patients asthmatiques sévères non contrôlés qui ne sont pas éligibles aux traitements biologiques disponibles.

En conclusion, cette étude met en lumière l'ampleur des besoins médicaux non couverts dans la population d'asthmatiques sévères, et montre ainsi la nécessité de développer de nouvelles alternatives thérapeutiques pour améliorer la prise en charge de cette population. 


\section{Références}

[1] Wenzel SE, Busse WW. Severe asthma: lessons from the Severe Asthma Research Program. J Allergy Clin Immunol 2007;119:14-21.

[2] Chung KF, Wenzel SE, Brozek JL, Bush A, Castro M, Sterk PJ, Adcock IM, Bateman ED, Bel EH, Bleecker ER, Boulet LP, Brightling C, Chanez P, Dahlen SE, Djukanovic R,Frey U,Gaga M, Gibson P, Hamid Q, Jajour NN, Mauad T, Sorkness RL, Teague WG. International ERS/ATS guidelines on definition, evaluation and treatment of severe asthma. Eur Respir $\mathbf{J}$ 2014;43:343-73.

[3] Hekking PP, Wener RR, Amelink M, Zwinderman AH, Bouvy ML, Bel EH. The prevalence of severe refractory asthma. J Allergy Clin Immunol 2015;135:896-902.

[4] Zeiger RS, Schatz M, Dalal AA, Qian L, Chen W, Ngor EW, Suruki RY, Kawatkar AA. Utilization and costs of severe uncontrolled asthma in a managed-care setting. J Allergy Clin Immunol Pract 2016;4:120-9.

[5] Haldar P, Pavord ID, Shaw DE, Berry MA, Thomas M, Brightling CE, Wardlaw AJ, Green RH. Cluster analysis and clinical asthma phenotypes. Am J Respir Crit Care Med 2008;178:218-24.

[6] Wenzel S. Severe asthma: from characteristics to phenotypes to endotypes. Clin Exp Allergy 2012;42:650-8.

[7] Moore WC, Meyers DA, Wenzel SE, Teague WG, Li H, Li X, D'Agostino R Jr, Castro M, Curran-Everett D, Fitzpatrick AM, Gaston B, Jarjour NN, Sorkness R, Calhoun WJ, Chung KF, Comhair SA, Dweik RA, Israel E, Peters SP, Busse WW, Erzurum SC, Bleecker ER. National Heart, Lung, and Blood Institute's Severe Asthma Research Program. Identification of asthma phenotypes using cluster analysis in the Severe Asthma Research Program. Am J Respir Crit Care Med 2010; 181:315-23. 
[8] Sekiya K, Nakatani E, Fukutomi Y, Kaneda H, Iikura M, Yoshida M, Takahashi K, Tomii K, Nishikawa M, Kaneko N, Sugino Y, Shinkai M, Ueda T, Tanikawa Y, Shirai T, Hirabayashi M, Aoki T, Kato T, Iizuka K, Homma S, Taniguchi M, Tanaka H. Severe or life-threatening asthma exacerbation: patient heterogeneity identified by cluster analysis. Clin Exp Allergy 2016;46:1043-55.

[9] Froidure A, Mouthuy J, Durham SR, Chanez P, Sibille Y, Pilette C. Asthma phenotypes and IgE responses. Eur Respir J 2016;47:304-19.

[10] Gras D, Chanez P. New sociology for better understanding severe eosinophilic asthma: introducing the SOCS family. Eur Respir J 2016;48:608-10.

[11]Pavord ID, Hilvering B, Shrimanker R. Emerging biologics in severe asthma. Immunol Allergy Clin North Am 2016;36:609-23.

[12]Fajt ML, Wenzel SE. Asthma phenotypes and the use of biologic medications in asthma and allergic disease: the next steps toward personalized care. J Allergy Clin Immunol 2015;135:299-310.

[13] Sposato B, Scalese M, Latorre M, Scichilone N, Matucci A, Milanese M, Masieri S, Rolla G, Steinhilber G, Rosati Y, Vultaggio A, Folletti I, Baglioni S, Bargagli E, Di Tomassi M, Pio R, Pio A, Maccari U, Maggiorelli C, Migliorini MG, Vignale L, Pulerà N, Carpagnano GE, Foschino Barbaro MP, Perrella A, Paggiaro PL. Effects of omalizumab in severe asthmatics across ages: A real life Italian experience. Respir Med 2016;119:141-9.

[14]Lugogo N, Domingo C, Chanez P, Leigh R, Gilson MJ, Price RG, Yancey SW, Ortega HG. Long-term efficacy and safety of mepolizumab in patients with severe eosinophilic asthma: A multi-center, open-label, phase IIIb study. Clin Ther 2016;38:2058-70.

[15] Maselli DJ, Velez MI, Rogers L. Reslizumab in the management of poorly controlled asthma: the data so far. J Asthma Allergy 2016;9:155-62. 
[16] Albers F, Müllerova H, Gunsoy NB, Shin JY, Nelsen LH, Bradford ES, Cockle SM, Suruki RY. Biologic treatment eligibility for real-world patients with severe asthma: the IDEAL study. J Asthma 2018;55:152-60

[17]Pradère P, Garcia G, Humbert M, Aubier M, Taillé C. Omalizumab : qu'avons-nous appris après 10 ans d'utilisation ? Rev Mal Respir 2016;33:117-127.

[18]Bel EH, Wenzel SE, Thompson PJ, Prazma CM, Keene ON, Yancey SW, Ortega HG, Pavord ID. SIRIUS Investigators. Oral glucocorticoid-sparing effect of mepolizumab in eosinophilic asthma. N Engl J Med 2014;371:1189-97.

[19] Ortega HG, Liu MC, Pavord ID, Brusselle GG, FitzGerald JM, Chetta A, Humbert M, Katz LE, Keene ON, Yancey SW, Chanez P; MENSA Investigators. Mepolizumab treatment in patients with severe eosinophilic asthma. N Engl J Med 2014;371:1198-207.

[20] Jones PW, Quirk FH, Baveystock CM, Littlejohns P. A self-complete measure of health status for chronic airflow limitation. The St. George's Respiratory Questionnaire. Am Rev Respir Dis $1992 ; 145: 1321-7$.

[21]Juniper EF, Buist AS, Cox FM, Ferrie PJ, King DR. Validation of a standardized version of the Asthma Quality of Life Questionnaire. Chest 1999;115:1265-70.

[22]EuroQol Group. EuroQol-a new facility for the measurement of health-related quality of life. Health Policy 1990;16:199-208.

[23] Reilly MC, Zbrozek AS, Dukes EM. The validity and reproducibility of a work productivity and activity impairment instrument. Pharmacoeconomics 1993;4:353-65.

[24]Bime C, Wei CY, Holbrook JT, Sockrider MM, Revicki DA, Wise RA. Asthma symptom utility index: reliability, validity, responsiveness, and the minimal important difference in adult asthmatic patients. J Allergy Clin Immunol 2012;130:1078-84. 
[25] Silkoff PE, Strambu I, Laviolette M, Singh D, FitzGerald JM, Lam S, Kelsen S, Eich A, Ludwig-Sengpiel A, Hupp GC, Backer V, Porsbjerg C, Girodet PO, Berger P, Leigh R, Kline JN, Dransfield M, Calhoun W, Hussaini A, Khatri S, Chanez P, Susulic VS, Barnathan ES, Curran M, Das AM, Brodmerkel C, Baribaud F, Loza MJ. Asthma characteristics and biomarkers from the Airways Disease Endotyping for Personalized Therapeutics (ADEPT) longitudinal profiling study. Respir Res 2015;16:142.

[26] The ENFUMOSA study group. The ENFUMOSA cross-sectional European multicentre study of the clinical phenotype of chronic severe asthma. European Network for Understanding Mechanisms of Severe Asthma. Eur Respir J 2003;22:470-7.

[27]Heaney LG, Brightling CE, Menzies-Gow A, Stevenson M, Niven RM; British Thoracic Society Difficult Asthma Network. Refractory asthma in the UK: cross-sectional findings from a UK multicentre registry.Thorax 2010;65:787-94.

[28] Chipps BE, Zeiger RS, Borish L, Wenzel SE, Yegin A, Hayden ML, Miller DP, Bleecker ER, Simons FE, Szefler SJ, Weiss ST, Haselkorn T; TENOR Study Group. Key findings and clinical implications from The Epidemiology and Natural History of Asthma: Outcomes and Treatment Regimens (TENOR) study. J Allergy Clin Immunol 2012;130:332-42.

[29] Kupczyk M, Haque S, Sterk PJ, Niżankowska-Mogilnicka E, Papi A, Bel EH, Chanez P, Dahlén B, Gaga M, Gjomarkaj M, Howarth PH, Johnston SL, Joos GF, Kanniess F, Tzortzaki E, James A, Middelveld RJ, Dahlén SE; BIOAIR investigators. Detection of exacerbations in asthma based on electronic diary data: results from the 1-year prospective BIOAIR study. Thorax 2013;68:611-8.

[30] Schleich F, Brusselle G, Louis R, Louis R, Vandenplas O, Michils A, Pilette C, Peche R, Manise M, Joos G. Heterogeneity of phenotypes in severe asthmatics. The Belgian Severe Asthma Registry (BSAR). Respir Med 2014;108:1723-32. 
[31]Price DB, Rigazio A, Campbell JD, Bleecker ER, Corrigan CJ, Thomas M, Wenzel SE, Wilson AM, Small MB, Gopalan G, Ashton VL, Burden A, Hillyer EV, Kerkhof M, Pavord ID. Blood eosinophil count and prospective annual asthma disease burden: a UK cohort study. Lancet Respir Med 2015;3:849-58.

[32] Mathew J, Aronow WS, Chandy D. Therapeutic options for severe asthma. Arch Med Sci 2012;8:589-97.

[33]Porsbjerg C, Menzies-Gow A. Co-morbidities in severe asthma: clinical impact and management. Respirology 2017;22:651-61.

[34] Moore WC, Bleecker ER, Curran-Everett D, Erzurum SC, Ameredes BT, Bacharier L, Calhoun WJ, Castro M, Chung KF, Clark MP, Dweik RA, Fitzpatrick AM, Gaston B, Hew M, Hussain I, Jarjour NN, Israel E, Levy BD, Murphy JR, Peters SP, Teague WG, Meyers DA, Busse WW, Wenzel SE; National Heart, Lung, Blood Institute's Severe Asthma Research Program. Characterization of the severe asthma phenotype by the National Heart, Lung, and Blood Institute's Severe Asthma Research Program. J Allergy Clin Immunol 2007;119:405-13.

[35]Nordon C, Grimaldi-Bensouda L, Pribil C, Nachbaur G, Amzal B, Thabut G, Marthan R, Aubier M, on the behalf of the COBRA study. Clinical and economic burden of severe asthma: a French cohort study. Europ Clin Immunol 2007;119:405-13.

[36] Avis CT NUCALA https://www.has-sante.fr/portail/upload/docs/evamed/CT14895_NUCALA_PIC_INS_Avis3_CT14895.pdf

[37] Avis CT CINQAERO https://www.has-sante.fr/portail/upload/docs/evamed/CT15839_CINQAERO_PIC_INS_Avis2_CT15839.pdf 
Légende des figures

Figure 1. Recrutement des patients constituant la cohorte française de l'étude IDEAL (schéma CONSORT) 
Tableau 1. Critères d'éligibilité aux trois traitements biologiques (mépolizumab, omalizumab, reslizumab) lors de la visite de l'étude

\begin{tabular}{|c|c|c|c|}
\hline \multirow[b]{2}{*}{ CRITERES } & \multicolumn{3}{|c|}{ 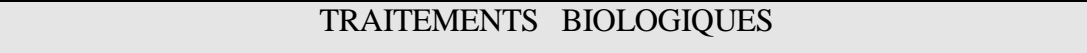 } \\
\hline & Mépolizumab & Omalizumab $^{\mathrm{a}}$ & Reslizumab \\
\hline $\begin{array}{l}\text { Nombre d'exacerbations } \\
\text { au cours des } 12 \text { derniers } \\
\text { mois nécessitant } \mathrm{CO} / \mathrm{ou} \\
\text { venue aux urgences /ou } \\
\text { hospitalisation }\end{array}$ & Au moins 2 & $\begin{array}{l}\text { Au moins } 2 \\
\text { ou au moins } 1 \\
\text { nécessitant une } \\
\text { hospitalisation }\end{array}$ & Au moins 1 \\
\hline Eosinophiles sanguins & $\begin{array}{l}\geq 150 \text { cellules } / \mu 1 \text { à } V 1 \text { ou } \\
\geq 300 \text { cellules/ } \mu 1 \text { au cours } \\
\text { des } 12 \text { derniers mois }\end{array}$ & Non applicable & $\begin{array}{l}\geq 400 \text { cellules } / \mu 1 \text { à } V 1 \text { ou } \\
\text { avant le début du } \\
\text { traitement }\end{array}$ \\
\hline $\begin{array}{l}\text { Taux d'IgE avant le début } \\
\text { du traitement par } \\
\text { omalizumab }\end{array}$ & Non applicable & $\begin{array}{l}\text { Taux d'IgE en fonction du } \\
\text { poids tel que décrit dans le } \\
\text { RCP européen }\end{array}$ & Non applicable \\
\hline Asthme allergique & Non applicable & $\begin{array}{l}\text { Test cutané ou RAST } \\
\text { positif }\end{array}$ & Non applicable \\
\hline Fonction respiratoire & Non applicable & VEMS $\leq 80 \%$ & $\begin{array}{l}\text { Réversibilité } \geq 12 \% \text { à } \\
\text { l'administration de SABA }\end{array}$ \\
\hline Score ACQ-5 (0-6) & Non applicable & $\geq 1,5$ & $\geq 1,5$ \\
\hline \multicolumn{4}{|c|}{$\begin{array}{l}\text { a avoir un traitement en cours avec l'omalizumab qualifie automatiquement le patient comme éligible à } \\
\text { l'omalizumab. } \\
\mathrm{CO}=\text { corticoïdes oraux ; ACQ-5 = questionnaire de contrôle de l'asthme en } 5 \text { items ; RAST = } \\
\text { radioallergosolbent } ; \mathrm{FEV}_{1}=\text { volume expiratoire maximal par seconde } ; \mathrm{SABA}=\text { béta2-agoniste de courte durée } \\
\text { d'action }\end{array}$} \\
\hline
\end{tabular}


Tableau 2. Caractéristiques démographiques et cliniques de la cohorte française de l'étude IDEAL selon le traitement en cours par omalizumab (OMA) lors de la visite de l'étude ( $\mathrm{N}=129)$

\begin{tabular}{|c|c|c|c|c|}
\hline Caractéristiques & $\begin{array}{l}\text { Cohorte mondiale } \\
\qquad(\mathrm{N}=670)[16]\end{array}$ & $\begin{array}{c}\text { Cohorte totale } \\
\text { française } \\
(\mathrm{N}=129)\end{array}$ & $\begin{array}{c}\text { Non en cours de } \\
\text { traitement par OMA } \\
(\mathrm{N}=86)\end{array}$ & $\begin{array}{c}\text { En cours de traitement } \\
\text { par OMA } \\
(\mathrm{N}=43)\end{array}$ \\
\hline \multicolumn{5}{|l|}{ Caractéristiques démographiques } \\
\hline Age ${ }^{a}$ (années) & $50,5 \pm 15,6$ & $52,8 \pm 15,9$ & $54,7 \pm 14,7$ & $48,9 \pm 17,5$ \\
\hline $\operatorname{Age}^{b}$ (années) & 51 [12-89] & $54[13-89]$ & $58[18-89]$ & $49[13-81]$ \\
\hline Femmes & $415(62 \%)$ & $82(64 \%)$ & $57(66 \%)$ & $25(58 \%)$ \\
\hline $\operatorname{IMC} C^{a}\left(\mathrm{~kg} / \mathrm{m}^{2}\right)$ & $29,9 \pm 7,4$ & $27,2 \pm 6,2$ & $27,2 \pm 6,5$ & $27,4 \pm 5,6$ \\
\hline \multicolumn{5}{|l|}{ Tabagisme } \\
\hline Actuel & $52(8 \%)$ & $15(12 \%)$ & $12(14 \%)$ & $3(7 \%)$ \\
\hline Ancien & $190(28 \%)$ & $38(29 \%)$ & $23(27 \%)$ & $15(35 \%)$ \\
\hline Aucun & $428(64 \%)$ & $76(59 \%)$ & $51(59 \%)$ & $25(58 \%)$ \\
\hline \multicolumn{5}{|l|}{ Caractéristiques cliniques } \\
\hline Comorbidités & $630(94 \%)$ & $112(87 \%)$ & $75(87 \%)$ & $37(86 \%)$ \\
\hline Troubles respiratoires & $476(71 \%)$ & $69(54 \%)$ & $50(58 \%)$ & $19(42 \%)$ \\
\hline Troubles du métabolisme & $340(51 \%)$ & $56(43 \%)$ & $38(44 \%)$ & $18(42 \%)$ \\
\hline Maladies cardiovasculaires & $241(36 \%)$ & $40(31 \%)$ & $29(34 \%)$ & $11(26 \%)$ \\
\hline Troubles du système nerveux & $222(33 \%)$ & $42(33 \%)$ & $29(34 \%)$ & $13(30 \%)$ \\
\hline Infections & $89(13 \%)$ & $19(15 \%)$ & $15(17 \%)$ & $4(9 \%)$ \\
\hline Autres & $334(50 \%)$ & $59(46 \%)$ & $38(44 \%)$ & $21(49 \%)$ \\
\hline \multicolumn{5}{|l|}{ Indice de Charlson ${ }^{c}$} \\
\hline 0 & $496(74 \%)$ & $95(74 \%)$ & $64(74,5 \%)$ & $31(72 \%)$ \\
\hline 1 & $114(17 \%)$ & $17(13 \%)$ & $12(14 \%)$ & $5(12 \%)$ \\
\hline 2 & $34(5 \%)$ & $9(7 \%)$ & $7(8 \%)$ & $2(5 \%)$ \\
\hline 3 & $14(2 \%)$ & $5(4 \%)$ & $1(1 \%)$ & $4(9 \%)$ \\
\hline$\geq 4$ & $12(2 \%)$ & $3(2 \%)$ & $2(2,5 \%)$ & $1(2 \%)$ \\
\hline \multicolumn{5}{|l|}{ Fonction respiratoire } \\
\hline VEMS prébronchodilatateur $(\mathrm{L})^{a}$ & $2,1 \pm 0,8$ & $2,1 \pm 0,9$ & $2,0 \pm 0,9$ & $2,2 \pm 0,8$ \\
\hline VEMS (\% valeur théorique) ${ }^{a}$ & $69 \pm 21$ & $70 \pm 24$ & $69 \pm 24$ & $72 \pm 23$ \\
\hline VEMS/CV ${ }^{\mathrm{a}}$ & $0,68 \pm 0,13$ & $0,68 \pm 0,13$ & $0,67 \pm 0,12$ & $0,69 \pm 0,14$ \\
\hline$\%$ réversibilité ${ }^{a}$ & $13 \pm 16$ & $9 \pm 13$ & $9 \pm 11$ & $10 \pm 17$ \\
\hline$\geq 12 \%$ réversibilité & $268(40 \%)$ & $32(25 \%)$ & $21(24 \%)$ & $11(26 \%)$ \\
\hline \multicolumn{5}{|l|}{ Caractéristiques de l'asthme } \\
\hline Antécédents familiaux d'asthme & $347(52 \%)$ & $60(47 \%)$ & $45(54 \%)$ & $15(35 \%)$ \\
\hline Ancienneté de l'asthme (années) ${ }^{a}$ & $26 \pm 17$ & $27 \pm 17$ & $31 \pm 18$ & $20 \pm 14$ \\
\hline \multicolumn{5}{|l|}{ Durée de l'asthme } \\
\hline$\geq 1$ et $<5$ ans & $62(9 \%)$ & $10(8 \%)$ & $3(3,5 \%)$ & $7(16 \%)$ \\
\hline$\geq 5$ et $<10$ ans & $74(11 \%)$ & $19(15 \%)$ & $13(15 \%)$ & $6(14 \%)$ \\
\hline$\geq 10$ et $<15$ ans & $72(12 \%)$ & $9(7 \%)$ & $4(5 \%)$ & $5(12 \%)$ \\
\hline$\geq 15$ et $<20$ ans & $74(11 \%)$ & $9(7 \%)$ & $5(6 \%)$ & $4(9 \%)$ \\
\hline$\geq 20$ et $<25$ ans & $57(8 \%)$ & $11(8 \%)$ & $9(10,5 \%)$ & $2(5 \%)$ \\
\hline$\geq 25$ ans & $331(49 \%)$ & $71(55 \%)$ & $52(60 \%)$ & $19(44 \%)$ \\
\hline Pas d'antécédents d'intubation ${ }^{d}$ & $616(92 \%)$ & $117(91 \%)$ & $79(92 \%)$ & $38(88 \%)$ \\
\hline Atopie (test RAST positif) & $416(62 \%)$ & $69(53,5 \%)$ & $41(48 \%)$ & $28(65 \%)$ \\
\hline \multicolumn{5}{|l|}{ Exacerbations $^{d}$} \\
\hline Nombre $^{\mathrm{a}}$ & $1,2 \pm 1,6$ & $1,5 \pm 1,9$ & $1,5 \pm 1,9$ & $1,5 \pm 2,0$ \\
\hline $\begin{array}{l}\geq 2 \text { avec } \mathrm{CO} \text { et/ou venue aux } \\
\text { urgences et/ou hospitalisation }\end{array}$ & $197(29 \%)$ & $47(36 \%)$ & $33(38 \%)$ & $14(33 \%)$ \\
\hline$\geq 1$ avec hospitalisation & $58(9 \%)$ & $15(12 \%)$ & $10(12 \%)$ & $5(12 \%)$ \\
\hline \multicolumn{5}{|l|}{ Taux d'éosinophiles } \\
\hline \multicolumn{5}{|l|}{ Au cours des 12 derniers mois } \\
\hline Non rapportés ${ }^{\mathrm{d}}$ & $441(66 \%)$ & $67(52 \%)$ & $47(55 \%)$ & $20(46,5 \%)$ \\
\hline Taux $<300$ cellules $/ \mu L^{d}$ & $123(18 \%)$ & $28(22 \%)$ & $19(22 \%)$ & $9(21,0 \%)$ \\
\hline
\end{tabular}




\begin{tabular}{|c|c|c|c|c|}
\hline Taux $\geq 300$ cellules $/ \mu L^{d}$ & $106(16 \%)$ & $34(26 \%)$ & $20(23 \%)$ & $14(32,5 \%)$ \\
\hline \multicolumn{5}{|l|}{ A la visite V1 } \\
\hline Taux $\geq 150$ cellules $/ \mu \mathrm{L}$ & $425(63 \%)$ & $85(66 \%)$ & $56(65 \%)$ & $29(67 \%)$ \\
\hline Taux $\geq 300$ cellules $/ \mu \mathrm{L}$ & $214(32 \%)$ & $44(34 \%)$ & $30(35 \%)$ & $14(33 \%)$ \\
\hline Taux $\geq 400$ cellules $/ \mu \mathrm{L}$ & $139(21 \%)$ & $29(22 \%)$ & $19(22 \%)$ & $10(23 \%)$ \\
\hline Taux $\geq 500$ cellules $/ \mu \mathrm{L}$ & $90(13 \%)$ & $16(12 \%)$ & $9(10,5 \%)$ & $7(16 \%)$ \\
\hline Taux IgE totales kU/L (à V1) & $155 \pm 1,6$ & $174 \pm 1,9$ & $99 \pm 1,8$ & $542 \pm 14$ \\
\hline Taux $<30$ & $99(15 \%)$ & $22(17 \%)$ & $21(24 \%)$ & $1(2 \%)$ \\
\hline$\geq 30$ et $<300$ & $326(49 \%)$ & $52(40 \%)$ & $40(47 \%)$ & $12(28 \%)$ \\
\hline$\geq 300$ et $<500$ & $102(15 \%)$ & $17(13 \%)$ & $11(13 \%)$ & $6(14 \%)$ \\
\hline$\geq 500$ et $<600$ & $20(3 \%)$ & $6(5 \%)$ & $3(3 \%)$ & $3(7 \%)$ \\
\hline$\geq 600$ et $<700$ & $19(3 \%)$ & $5(4 \%)$ & $4(5 \%)$ & $1(2 \%)$ \\
\hline$\geq 700$ et $<800$ & $13(2 \%)$ & $2(2 \%)$ & - & $2(5 \%)$ \\
\hline$\geq 800$ et $<900$ & $10(1,5 \%)$ & $3(2 \%)$ & $1(1 \%)$ & $2(5 \%)$ \\
\hline$\geq 900$ et $<1000$ & $8(1 \%)$ & $3(2 \%)$ & $1(1 \%)$ & $2(5 \%)$ \\
\hline$\geq 1000$ et $<1200$ & $14(2 \%)$ & - & - & - \\
\hline$\geq 1200$ et $<1500$ & $16(2 \%)$ & $4(3 \%)$ & $1(1 \%)$ & $3(7 \%)$ \\
\hline$>1500$ & $43(6,5 \%)$ & $15(12 \%)$ & $4(5 \%)$ & $11(25 \%)$ \\
\hline \multicolumn{5}{|l|}{ Traitements } \\
\hline $\mathrm{CO}$ en traitement de fond (actuel) & $94(14 \%)$ & $31(24 \%)$ & $23(27 \%)$ & $8(19 \%)$ \\
\hline \multicolumn{5}{|l|}{$O M A$} \\
\hline Antécédent de traitement ${ }^{\mathrm{d}}$ & $183(27 \%)$ & $47(36 \%)$ & $6(7 \%)$ & $41(95 \%)$ \\
\hline Arrêt du traitement & $15(8 \%)$ & $5(11 \%)$ & $5(83 \%)$ & $0(0 \%)$ \\
\hline Manque d'efficacité & $5(33 \%)$ & $1(20 \%)$ & $1(20 \%)$ & - \\
\hline Effets secondaires & $4(27 \%)$ & $2(40 \%)$ & $2(40 \%)$ & - \\
\hline Autres raisons & $6(40 \%)$ & $2(40 \%)$ & $2(40 \%)$ & - \\
\hline Durée du traitement $\mathrm{OMA}^{\mathrm{a}}$, mois & $34,4 \pm 29,7$ & $26,5 \pm 21,4$ & $30,8 \pm 26,3$ & $25,9 \pm 20,9$ \\
\hline \multicolumn{5}{|l|}{ Résultats des auto-questionnaires ${ }^{\mathrm{a}}(*)$} \\
\hline Score ACQ-5 (0-6) & $2,08 \pm 1,26(670)^{*}$ & $2,26 \pm 1,43(129)^{*}$ & $2,19 \pm 1,37(86)^{*}$ & $2,40 \pm 1,54(43)^{*}$ \\
\hline Score SGRQ(0-100) & $42,2 \pm 20,0(651)^{*}$ & $45,8 \pm 21,7(119)^{*}$ & $45,9 \pm 21,9(78)^{*}$ & $45,6 \pm 21,4(41)^{*}$ \\
\hline Score AQLQ (0-6) & $4,6 \pm 1,3(624)^{*}$ & $4,4 \pm 1,3(112)^{*}$ & $4,5 \pm 1,3(74)^{*}$ & $4,3 \pm 1,3(38)^{*}$ \\
\hline Score EQ-5D-5L(0-1) & $0,796 \pm 0,216(669)^{*}$ & $0,725 \pm 0,259(128)^{*}$ & $0,725 \pm 0,2496(85)^{*}$ & $0,725 \pm 0,280(43)^{*}$ \\
\hline Score EQ-EVA $(0-100)$ & $62,2 \pm 21,56(669)^{*}$ & $62,2 \pm 21,56(129)^{*}$ & $62,7 \pm 22,5(86)^{*}$ & $61,2 \pm 19,76(43)^{*}$ \\
\hline Score ASUI(0-1) & $0,68 \pm 0,24(628)^{*}$ & $0,63 \pm 0,26(123)^{*}$ & $0,64 \pm 0,27(81)^{*}$ & $0,61 \pm 0,26(42)^{*}$ \\
\hline \multicolumn{5}{|l|}{ Score WPAI $(0-100)$ : } \\
\hline Impact sur la productivité au travail & $23,5 \pm 27,0(328)^{*}$ & $25,1 \pm 28,8(43)^{*}$ & $20,2 \pm 25,8(30)^{*}$ & $36,5 \pm 33,0(13)^{*}$ \\
\hline $\begin{array}{l}\text { Impact sur la capacité à effectuer les } \\
\text { activités quotidiennes }\end{array}$ & $37,3 \pm 28,8(667)^{*}$ & $40,1 \pm 28,4(126)^{*}$ & $39,0 \pm 28,7(83)^{*}$ & $42,1 \pm 28,2(43)^{*}$ \\
\hline
\end{tabular}

Les données sont exprimées en $\mathrm{n}(\%),{ }^{\mathrm{a}}$ moyenne \pm écart-type, ${ }^{\mathrm{b}}$ médiane $] 25^{\mathrm{e}}-75^{\mathrm{e}}$ percentile], ${ }^{\mathrm{c}}$ Score de comorbidité prédictif de survie, ${ }^{\mathrm{d}}$ au cours des 12 derniers mois, ${ }^{\mathrm{e}}$ Test RAST (radioallergosorbent test), ${ }^{\mathrm{f}}$ moyenne géométrique $\pm \log$ de l'écart type, *Nombre de réponses aux questionnaires

ACQ-5 = questionnaire de contrôle de l'asthme en 5 items ; AQLQ = questionnaire standardisé de qualité de vie $; \mathrm{ASUI}=$ «Asthma symptom utility index »; $\mathrm{CO}=$ corticoïdes oraux ; $\mathrm{CV}=$ capacité vitale ; EQ5D-5L = questionnaire standardisé de qualité de vie $;$ EQ-EVA = échelle analogique visuelle ; IMC = index de masse corporelle ; OMA = omalizumab ; SGRQ = questionnaire respiratoire de Saint Georges ; $\mathrm{VEMS}=$ volume expiratoire maximale par seconde; WPAI $=$ «Work productivity and activity impairment » 
Tableau 3. Eligibilité aux trois traitements biologiques (mépolizumab, omalizumab, reslizumab) selon le traitement en cours par omalizumab lors de la visite de l'étude ( $\mathrm{N}=129)$

\begin{tabular}{|c|c|c|c|c|c|c|}
\hline \multirow[t]{2}{*}{ Eligibilité à } & \multicolumn{2}{|r|}{$\begin{array}{l}\text { Cohorte totale } \\
\qquad(\mathrm{N}=129)\end{array}$} & \multicolumn{2}{|c|}{$\begin{array}{c}\text { Non en cours } \\
\text { de traitement par OMA } \\
(\mathrm{N}=86)\end{array}$} & \multicolumn{2}{|c|}{$\begin{array}{c}\text { En cours } \\
\text { de traitement par OMA } \\
(\mathrm{N}=43)\end{array}$} \\
\hline & $\mathrm{n}$ & $\%($ IC $95 \%)$ & $\mathrm{n}$ & $\%(\mathrm{IC} 95 \%)$ & $\mathrm{n}$ & $\%($ IC $95 \%)$ \\
\hline Mépolizumab & 35 & $\begin{array}{c}27,1 \% \\
(19,7 \%-35,7 \%)\end{array}$ & 25 & $\begin{array}{c}29,1 \% \\
(19,8 \%-39,9 \%)\end{array}$ & 10 & $\begin{array}{c}23,3 \% \\
(11,8 \%-38,6 \%)\end{array}$ \\
\hline $\begin{array}{l}\text { Omalizumab } \\
\text { (OMA) }\end{array}$ & 52 & $\begin{array}{c}40,3 \% \\
(31,8 \%-49,3 \%)\end{array}$ & 9 & $\begin{array}{c}10,5 \% \\
(4,9 \%-18,9 \%)\end{array}$ & 43 & $100 \%$ \\
\hline Reslizumab & 3 & $\begin{array}{c}2,3 \% \\
(0,5 \%-6,6 \%)\end{array}$ & 3 & $\begin{array}{c}3,5 \% \\
(0,7 \%-9,9 \%)\end{array}$ & 0 & - \\
\hline
\end{tabular}


Tableau 4. Caractéristiques de la cohorte française de l'étude IDEAL selon l'éligibilité aux traitements biologiques (omalizumab, mépolizumab, reslizumab) $(\mathrm{N}=129)$

\begin{tabular}{|c|c|c|c|}
\hline Caractéristiques & $\begin{array}{c}\text { Eligible au } \\
\text { mépolizumab } \\
(\mathrm{N}=35)\end{array}$ & $\begin{array}{c}\text { Eligible à } \\
\text { l'omalizumab } \\
(\mathrm{N}=52)\end{array}$ & $\begin{array}{c}\text { Eligible au } \\
\text { reslizumab } \\
(\mathrm{N}=3)\end{array}$ \\
\hline \multicolumn{4}{|l|}{ Caractéristiques démographiques } \\
\hline $\operatorname{Age}^{a}$ (années) & $49,6 \pm 14,2$ & $49,4 \pm 16,4$ & $57,1 \pm 14,1$ \\
\hline $\operatorname{Age}^{b}$ (années) & $52[14-74]$ & $49[13-81]$ & $59[42-70]$ \\
\hline Femmes, \% & $24(69 \%)$ & $32(62 \%)$ & $2(67 \%)$ \\
\hline $\operatorname{IMC}^{a}\left(\mathrm{~kg} / \mathrm{m}^{2}\right)$ & $28,4 \pm 7,0$ & $27,3 \pm 5,5$ & $28,7 \pm 0,4$ \\
\hline \multicolumn{4}{|l|}{ Tabagisme } \\
\hline Actuel & $4(11 \%)$ & $6(12 \%)$ & 0 \\
\hline Ancien & $10(29 \%)$ & $17(33 \%)$ & $1(33 \%)$ \\
\hline Aucun & $21(60 \%)$ & $29(56 \%)$ & $2(67 \%)$ \\
\hline \multicolumn{4}{|l|}{ Caractéristiques cliniques } \\
\hline Comorbidités & $32(91 \%)$ & $46(89 \%)$ & $3(100 \%)$ \\
\hline Troubles respiratoires & $18(51 \%)$ & $25(48 \%)$ & $2(67 \%)$ \\
\hline Troubles du métabolisme & $17(49 \%)$ & $23(44 \%)$ & $2(67 \%)$ \\
\hline Maladies cardiovasculaires & $9(26 \%)$ & $13(25 \%)$ & $1(33 \%)$ \\
\hline Troubles du système nerveux & $15(43 \%)$ & $18(35 \%)$ & $1(33 \%)$ \\
\hline Sinusites & $8(23 \%)$ & $6(12 \%)$ & $2(67 \%)$ \\
\hline \multicolumn{4}{|l|}{ Indice de Charlson } \\
\hline 0 & $23(66 \%)$ & $35(67 \%)$ & $3(100 \%)$ \\
\hline 1 & $5(14 \%)$ & $6(12 \%)$ & 0 \\
\hline 2 & $4(11 \%)$ & $6(12 \%)$ & 0 \\
\hline 3 & $2(6 \%)$ & $4(8 \%)$ & 0 \\
\hline$\geq 4$ & $1(3 \%)$ & $1(2 \%)$ & 0 \\
\hline \multicolumn{4}{|l|}{ Fonction respiratoire } \\
\hline VEMS prébronchondilatateur ${ }^{\mathrm{a}}(\mathrm{L})$ & $1,8 \pm 0,8$ & $2,1 \pm 0,8$ & $1,3 \pm 0,6$ \\
\hline VEMS (\% valeur théorique) $)^{\mathrm{a}}$ & $60,3 \pm 23,0$ & $69,5 \pm 22,7$ & $42,2 \pm 11,6$ \\
\hline $\mathrm{VEMS} / \mathrm{CV}^{\mathrm{a}}$ & $0,65 \pm 0,14$ & $0,68 \pm 0,13$ & $0,55 \pm 0,17$ \\
\hline$\%$ réversibilité ${ }^{\mathrm{a}}$ & $13,9 \pm 18,5$ & $10,8 \pm 15,7$ & $26,4 \pm 13,1$ \\
\hline$\geq 12 \%$ réversibilité & $12(34 \%)$ & $16(31 \%)$ & $3(100 \%)$ \\
\hline \multicolumn{4}{|l|}{ Caractéristiques de l'asthme } \\
\hline Antécédents familiaux d'asthme & $12(36 \%)$ & $22(43 \%)$ & $1(50 \%)$ \\
\hline Ancienneté de l'asthme' (années) ${ }^{a}$ & $24,4 \pm 16,8$ & $22,9 \pm 15,2$ & $16,7 \pm 17,7$ \\
\hline \multicolumn{4}{|l|}{ Durée de l'asthme } \\
\hline$\geq 1$ et $<5$ ans & $2(6 \%)$ & $7(14 \%)$ & 0 \\
\hline$\geq 5$ et $<10$ ans & $7(20 \%)$ & $6(11 \%)$ & $2(67 \%)$ \\
\hline$\geq 10$ et $<15$ ans & $4(11 \%)$ & $6(11 \%)$ & 0 \\
\hline$\geq 15$ et $<20$ ans & $4(11 \%)$ & $5(10 \%)$ & 0 \\
\hline$\geq 20$ et $<25$ ans & $3(9 \%)$ & $2(4 \%)$ & 0 \\
\hline$\geq 25$ ans & $15(43 \%)$ & $26(50 \%)$ & $1(33 \%)$ \\
\hline Pas d'antécédents d'intubation ${ }^{a}$ & $31(89 \%)$ & $47(90 \%)$ & $2(67 \%)$ \\
\hline Atopie (test RAST positif) ${ }^{d}$ & $18(51 \%)$ & $37(71 \%)$ & $1(33 \%)$ \\
\hline \multicolumn{4}{|l|}{ Exacerbations $^{c}$} \\
\hline Nombre $^{\mathrm{a}}$ & $3,8 \pm 2,1$ & $1,8 \pm 2,0$ & $6,0 \pm 3,6$ \\
\hline $\begin{array}{l}\geq 2 \text { avec } \mathrm{CO} \text { et/ou venue aux } \\
\text { urgences et/ou hospitalisation }\end{array}$ & $35(100 \%)$ & $23(44 \%)$ & $3(100 \%)$ \\
\hline$\geq 1$ avec hospitalisation & $9(26 \%)$ & $9(18 \%)$ & $0(0 \%)$ \\
\hline \multicolumn{4}{|l|}{ Taux d'éosinophiles } \\
\hline \multicolumn{4}{|l|}{ Au cours des 12 derniers mois } \\
\hline Non rapportés ${ }^{c}$ & $10(29 \%)$ & $22(42 \%)$ & 0 \\
\hline Taux $<300$ cellules $/ \mu \mathrm{L}^{\mathrm{c}}$ & $5(14 \%)$ & $13(25 \%)$ & $1(33 \%)$ \\
\hline Taux $\geq 300$ cellules $/ \mu \mathrm{L}^{\mathrm{c}}$ & $20(57 \%)$ & $17(33 \%)$ & $2(67 \%)$ \\
\hline
\end{tabular}




\begin{tabular}{|c|c|c|c|}
\hline \multicolumn{4}{|l|}{ A la visite $V 1$} \\
\hline Taux $\geq 150$ cellules $/ \mu \mathrm{L}$ & $28(80 \%)$ & $35(67 \%)$ & $3(100 \%)$ \\
\hline Taux $\geq 300$ cellules $/ \mu \mathrm{L}$ & $11(31,5 \%)$ & $15(29 \%)$ & $3(100 \%)$ \\
\hline Taux $\geq 400$ cellules $/ \mu \mathrm{L}$ & $8(23 \%)$ & $10(19 \%)$ & $3(100 \%)$ \\
\hline Taux $\geq 500$ cellules/ $\mu \mathrm{L}$ & $5(14 \%)$ & $7(13,5 \%)$ & $1(33 \%)$ \\
\hline Taux d' IgE totales kU/L (à V1) ${ }^{\mathrm{e}}$ & $177 \pm 1,8$ & $443 \pm 1,4$ & $61 \pm 1,8$ \\
\hline \multicolumn{4}{|l|}{ Traitements } \\
\hline CO en traitement de fond (actuel) & $9(26 \%)$ & $11(21 \%)$ & $0(0 \%)$ \\
\hline$O M A$ & $11(31 \%)$ & $42(81 \%)$ & $0(0 \%)$ \\
\hline Antécédent de traitement ${ }^{c}$ & $11(31 \%)$ & $42(81 \%)$ & 0 \\
\hline Echec au traitement & $2(18 \%)$ & $1(2 \%)$ & 0 \\
\hline Manque d'efficacité & - & - & - \\
\hline Effets secondaires & $1(50 \%)$ & $1(100 \%)$ & - \\
\hline Autres raisons & $1(50 \%)$ & - & - \\
\hline Durée du traitement OMA (mois) & $29,6 \pm 22,5$ & $25,3 \pm 20,9$ & 0 \\
\hline \multicolumn{4}{|l|}{ Résultats des auto-questionnaires ${ }^{\mathrm{a}}$} \\
\hline Score ACQ-5(0-6) & $2,8 \pm 1,5$ & $2,6 \pm 1,5$ & $3,4 \pm 0,5$ \\
\hline Score SGRQ $(0-100)$ & $54,1 \pm 20,9$ & $48,3 \pm 22,4$ & $56,6 \pm 23,4$ \\
\hline Score AQLQ $(0-6)$ & $4,2 \pm 1,3$ & $4,1 \pm 1,32$ & $4,2 \pm 1,0$ \\
\hline Score EQ-5D-5L $(0-1)$ & $0,672 \pm 0,278$ & $0,696 \pm 0,287$ & $0,655 \pm 0,252$ \\
\hline Score EQ-EVA $(0-100)$ & $56,7 \pm 20,0$ & $57,0 \pm 21,3$ & $65,0 \pm 8,7$ \\
\hline Score ASUI (0-100) & $0,528 \pm 0,266$ & $0,576 \pm 0,266$ & $0,403 \pm 0,159$ \\
\hline \multicolumn{4}{|l|}{ Score WPAI $(0-100)$ : } \\
\hline Impact sur la capacité à travailler & $33,0 \pm 26,5(10)^{*}$ & $36,3 \pm 31,7(14)^{*}$ & $40,0(1)^{*}$ \\
\hline $\begin{array}{l}\text { Impact sur la capacité à assurer les activités } \\
\text { habituelles }\end{array}$ & $47,9 \pm 28,5(33)^{*}$ & $44,9 \pm 28,1(51)^{*}$ & $50,0 \pm 10,0(3)^{*}$ \\
\hline
\end{tabular}

Les données sont exprimées en $\mathrm{n}(\%),{ }^{\mathrm{a}}$ moyenne \pm écart-type, ${ }^{\mathrm{b}}$ médiane $25^{\mathrm{e}}-75^{\mathrm{e}}$ percentile], ${ }^{\mathrm{c}}$ au cours des 12 derniers mois, ${ }^{\mathrm{d}}$ Test RAST, ${ }^{\mathrm{e}}$ moyenne géométrique \pm log de l'écart type, $*$ Nombre de réponses aux questionnaires

ACQ-5 = questionnaire de contrôle de l'asthme en 5 items ; AQLQ = questionnaire standardisé de qualité de vie ; ASUI $=$ «Asthma symptom utility index »; $\mathrm{CO}=$ corticoïdes oraux ; CV = capacité vitale ; EQ$5 \mathrm{D}-5 \mathrm{~L}=$ questionnaire standardisé de qualité de vie $; \mathrm{EQ}-\mathrm{EVA}=$ échelle analogique visuelle $;$ OMA = omalizumab ; sem = semaine ; SGRQ = questionnaire respiratoire de Saint Georges ; VEMS= volume expiratoire maximale par seconde ; WPAI $=$ « Work productivity and activity impairment » 
